\title{
Water Dielectric Effects in Planar Confinement
}

\author{
Alexander Schlaich, ${ }^{1, *}$ Ernst W. Knapp, ${ }^{2}$ and Roland R. Netz ${ }^{1, \dagger}$ \\ ${ }^{1}$ Fachbereich Physik, Freie Universität Berlin, Arnimallee 14, 14195 Berlin, Germany \\ ${ }^{2}$ Fachbereich Chemie, Freie Universität Berlin, Fabeckstraße 36a, 14195 Berlin, Germany \\ (Received 14 May 2016; revised manuscript received 10 June 2016; published 19 July 2016)
}

\begin{abstract}
We investigate the dielectric profile of water confined between two planar polar walls using atomistic molecular dynamics simulations. For a water slab thickness below $1 \mathrm{~nm}$ the dielectric response is highly asymmetric: while the parallel component slightly increases compared to bulk, the perpendicular one decreases drastically due to anticorrelated polarization of neighboring water molecules. We demonstrate the importance of the dielectric contribution due to flexible polar headgroups and derive an effective dielectric tensorial box model suitable for coarse-grained electrostatic modeling.
\end{abstract}

DOI: 10.1103/PhysRevLett.117.048001

The water structure at surfaces has a profound influence on wetting properties, adhesion between surfaces, and adsorption of ions, molecular solutes and macromolecules [1-4]. The understanding of hydration and hydrophobic interactions between surfaces has been possible by groundbreaking experimental techniques [5-7] and more recently been advanced by computer simulations that include explicit water [8-12].

Most biologically or technologically relevant surfaces such as lipid membranes [13], mineral surfaces, and colloids [14], bear surface charges. Electrostatic interactions in aqueous solution are strongly influenced by water's dielectric properties. The large relative dielectric constant of bulk water of $\varepsilon_{\text {bulk }}=80$ means that Coulomb interactions in water are reduced down to roughly 1 percent compared to vacuum. Stern's groundbreaking analysis of experimental surface capacitance data demonstrated that at a solid-water interface a thin interfacial layer exists with a dielectric constant much reduced compared to the bulk water value [15]. Although this interfacial layer has a thickness of only about a tenth of a nanometer, its effects on the surface capacitance as well as electrokinetic transport properties such as zeta potentials and surface conductivities are significant [16].

The interaction between charged particles and surfaces across aqueous solution is standardly modeled by continuum theories and solvent-implicit simulations assuming constant dielectric properties in the water slab [17-20]. Clearly, any modification of the water dielectric constant as the surfaces approach would strongly modify the electrostatic intersurface interactions.

Whereas the bulk dielectric response of polar fluids is well understood [21-24], atomistic simulations as well as analytic theories confirmed that it is strongly modified at interfaces [25-28]. For a planar interface, the dielectric response becomes anisotropic and can be described by two local profiles, $\varepsilon_{\|}(z)$ and $\varepsilon_{\perp}(z)$. The perpendicular component is particularly relevant and has been used to predict the surface zeta potential and capacitance in quantitative agreement with experimental data [29]. The effect of confinement on the dielectric constant, however, is highly relevant but far from settled [3]. Previous simulation studies suggested a reduced dielectric constant in confinement [30-33]; however, some of these studies were debated because of ill-defined boundary conditions [34,35].

In this Letter we extract the water dielectric properties between two soft polar model surfaces using extensive molecular dynamics (MD) simulations. Both parallel and perpendicular dielectric components reach the bulk value for a water slab thickness larger than about $1 \mathrm{~nm}$, for a smaller thickness the parallel dielectric component is enhanced while the perpendicular component is drastically reduced. This symmetry breaking is solely due to collective polarization effects: The parallel polarization correlations between neighboring water molecules is slightly enhanced, while the perpendicular dipoles of neighboring water molecules are predominantly anticorrelated, leading to a negative collective contribution. We also show that the orientational freedom of polar headgroups adds significantly to the local dielectric constant. We cast our results in the form of a tensorial dielectric box model which can be straightforwardly implemented in future coarse-grained modeling of the interaction between charged surfaces and electrokinetic effects in confinement [36,37].

Linear response theory.-We write the change of the dielectric displacement field $\Delta \vec{D}(\vec{r})$ at position $\vec{r}$ due to the local change of the electric field $\Delta \vec{E}(\vec{r})$ as an integral over the nonlocal dielectric response tensor $\stackrel{\leftrightarrow}{\varepsilon}_{n l}$,

$$
\Delta \vec{D}(\vec{r})=\varepsilon_{0} \int \overleftrightarrow{\varepsilon}_{n l}\left(\vec{r}, \vec{r}^{\prime}\right) \cdot \Delta \vec{E}\left(\vec{r}^{\prime}\right) d \vec{r}^{\prime}
$$

For planar symmetry in the $x y$ directions, all fields and response functions only depend on the $z$ coordinate. Furthermore, the static Maxwell relation $\vec{\nabla} \times \Delta \vec{E}(z)=0$ shows that the parallel electric field $\Delta E_{\|}$is constant and thus Eq. (1) simplifies to 


$$
\Delta D_{\|}(z)=\varepsilon_{0} \varepsilon_{\|}(z) \Delta E_{\|},
$$

where we define the local parallel response as $\varepsilon_{\|}(z)=\int \varepsilon_{n l}^{\|}\left(z, z^{\prime}\right) d z^{\prime}$. Similarly, the Maxwell relation in the absence of free charges $\vec{\nabla} \cdot \Delta \vec{D}(z)=0$ shows that the perpendicular displacement field $\Delta D_{\perp}$ is constant which leads to the perpendicular local response relation

$$
\Delta E_{\perp}(z)=\varepsilon_{0}^{-1} \varepsilon_{\perp}^{-1}(z) \Delta D_{\perp} .
$$

We define parallel and perpendicular polarization correlation functions as

$$
c_{\alpha}(z)=\left\langle m_{\alpha}(z) M_{\alpha}\right\rangle-\left\langle m_{\alpha}(z)\right\rangle\left\langle M_{\alpha}\right\rangle,
$$

with $m_{\alpha}(z)$ being the laterally averaged polarization density at position $z$ and $\alpha=\perp, \|$. The total polarization follows by an integral over the entire simulation box height, $M_{\alpha}=A \int_{-L_{z} / 2}^{L_{z} / 2} d z m_{\alpha}(z)$, where the lateral simulation box area is $A=L_{x} L_{y}$.

Using the fluctuation-dissipation theorem, $\varepsilon_{\|}(z)$ is related to polarization correlations via $[25,26]$

$$
\varepsilon_{\|}(z)=1+\frac{c_{\|}(z)}{\varepsilon_{0} k_{B} T}
$$

where $k_{B} T$ is the thermal energy and $\varepsilon_{0}$ denotes the vacuum permittivity. In contrast, the inverse perpendicular dielectric profile for a periodic system follows as [38]

$$
\varepsilon_{\perp}^{-1}(z)=1-\frac{c_{\perp}(z)}{\varepsilon_{0} k_{B} T+C_{\perp} / V},
$$

where $V=A L_{z}$ is the simulation box volume and the variance of the total polarization is denoted as $C_{\alpha}=A \int_{-L_{z} / 2}^{L_{z} / 2} d z c_{\alpha}(z)$. Note that for a homogeneous system we have $c_{\perp}(z)=C_{\perp} / V$ and thus $\varepsilon_{\perp}=$ $1+c_{\perp} /\left(\varepsilon_{0} k_{B} T\right)$, in full analogy to the parallel component in Eq. (5) and Ref. [39]. However, in the general interfacial scenario, where $c_{\perp}(z)$ depends on the coordinate $z$, the parallel and perpendicular expressions Eqs. (5) and (6) are intrinsically different, as we will discuss in detail.

MD simulations. - The slab between two planar surfaces consisting of 100 polar decanol molecules each is filled with $N_{w}=170$ up to $19752 \mathrm{SPC} / \mathrm{E}$ water molecules [40] (see Fig. 1 for a snapshot). The SPC/E model represents bulk water dielectric properties quite well [41]. Force-field parameters are based on GROMOS53A6 [42], decanol hydroxyl groups are represented in atomistic detail, $\mathrm{CH}_{2}$ and $\mathrm{CH}_{3}$ groups as united atoms. The repulsion between head-group oxygens is increased to reduce intrasurface hydrogen bonding [43]. To avoid slow reorientation events, we restrain all decanols on the second $\mathrm{CH}_{2}$ group counting from the $\mathrm{OH}$ headgroup with force constants $k_{x}=k_{y}=$ $500 \mathrm{~kJ} /\left(\mathrm{mol} \mathrm{nm}^{2}\right)$ and $k_{z}=10 \mathrm{~kJ} /\left(\mathrm{mol} \mathrm{nm}^{2}\right)$ and the

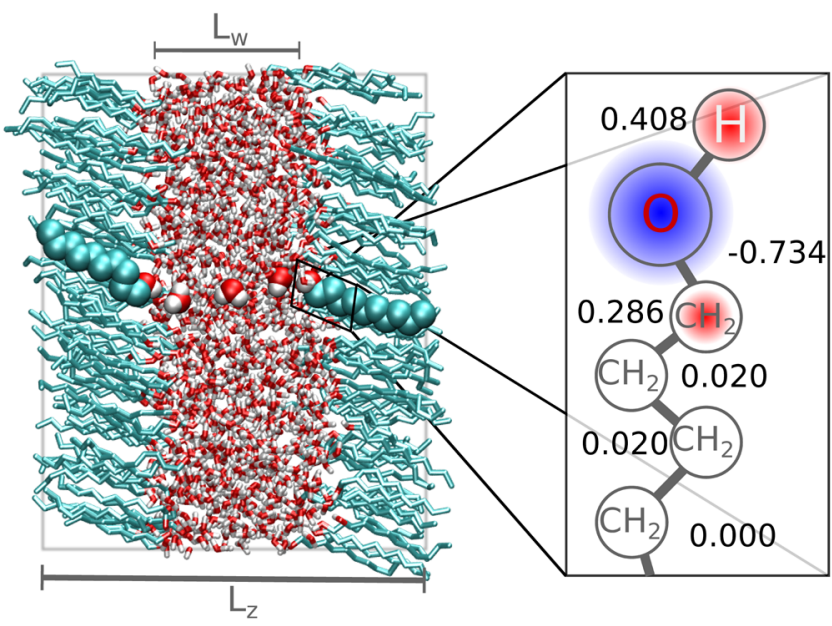

FIG. 1. Simulation snapshot for a water layer thickness $L_{w}=1.5 \mathrm{~nm}$. Selected molecules are shown using van der Waals spheres. Each monolayer consists of 100 decanol molecules. The inset on the right shows the distribution of partial charges on the flexible polar headgroups.

terminal $\mathrm{CH}_{3}$ group with $k_{x}=k_{y}=5 \mathrm{~kJ} /\left(\mathrm{mol} \mathrm{nm}^{2}\right)$ on a centered rectangular lattice with a lateral area per headgroup of $A / 100=0.234 \mathrm{~nm}^{2}$. This corresponds to the tensionless state in vacuum with a decanol tilt angle of $30^{\circ}$. Simulations are performed using version 5.0 of the GROMACS simulation package [44] at $T=300 \mathrm{~K}$ with periodic boundary conditions and analyzed using the MDAnalysis package [45].

In analogy to the experimental definition [46], we define the water layer thickness $L_{w}$ using the bulk molecular water volume $v_{w}=0.0304 \mathrm{~nm}^{3}$ and the number of water molecules $N_{w}$ as $L_{w}=N_{w} v_{w} / A$. For small separations $L_{w}<3 \mathrm{~nm}$, simulations are done in the $N_{w} A L_{z} T$ ensemble at constant volume $V=A L_{z}$ and the number of water molecules $N_{w}$ is adjusted via thermodynamic extrapolation to yield a constant chemical potential [11,47]. For $L_{w}>3 \mathrm{~nm}$, the interaction pressure is negligible and we use the $N_{w} A P_{z} T$ ensemble at fixed vertical pressure $P_{z}=1$ bar. Lennard Jones interactions are truncated at $r_{c}=0.9 \mathrm{~nm}$, for the electrostatic interactions the particle mesh Ewald method [57] is employed with a real-space cutoff $r_{c}=0.9 \mathrm{~nm}$. Simulations for $L_{w}<8 \mathrm{~nm}$ have a length of $1 \mu \mathrm{s}$, trajectories at larger separations are obtained for at least $100 \mathrm{~ns}$.

Dielectric profiles.-We analyze the water and decanol polarization contributions separately according to

$$
m_{\alpha}(z)=m_{\alpha}^{w}(z)+m_{\alpha}^{\mathrm{dec}}(z) .
$$

The perpendicular polarization profile is calculated via integration of the charge density $\rho(z)$ according to $m_{\perp}(z)=-\int_{-L_{z} / 2}^{z} \rho\left(z^{\prime}\right) d z^{\prime}$, the parallel polarization profile is calculated from the boundary polarization charge distribution [47,58]. Figure 2(a) shows the water, decanol, and the total mass density profiles $\rho_{m}(z)$ for $L_{w}=1.5 \mathrm{~nm}$ and 
demonstrates that water density oscillations (blue line) are absent as expected for our relatively soft surfaces $[43,59,60]$. The total mass density (black line) is rather uniform throughout the system. The vertical gray lines denote the Gibbs dividing surface positions, $z_{\mathrm{GDS}}$, which thermodynamically define the surface positions and follow from our definition of the water slab thickness as $z_{\mathrm{GDS}}= \pm L_{w} / 2$. The charge density profile in Fig. 2(b) reveals that the main contribution comes from the decanols (red line) which is due to the pronounced orientation of the headgroups with respect to the surface normal.

The parallel dielectric data in Fig. 2(c) demonstrate that the headgroups (red line) significantly contribute and produce a maximum in the total dielectric profile (black line) of $\varepsilon_{\|}=75$ close to the interface position, slightly larger than the bulk value $\varepsilon_{\text {bulk }}=70.0 \pm 0.2$ (denoted by a horizontal broken line, determined independently from bulk simulations and consistent with literature values for SPC/E water $[41,61])$.

In Fig. 2(d) we show $1-\varepsilon_{\perp}^{-1}(z)$ which according to Eq. (6) is proportional to the perpendicular polarization correlations $c_{\perp}(z)$ and thus can be decomposed into the water and decanol contributions. We see that headgroups (red line) contribute even more than in the parallel direction and that $1-\varepsilon_{\perp}^{-1}(z)>1$ inside the headgroup region. This means that the perpendicular dielectric profile $\varepsilon_{\perp}(z)$, shown in Fig. 2(e), exhibits poles close to the surface

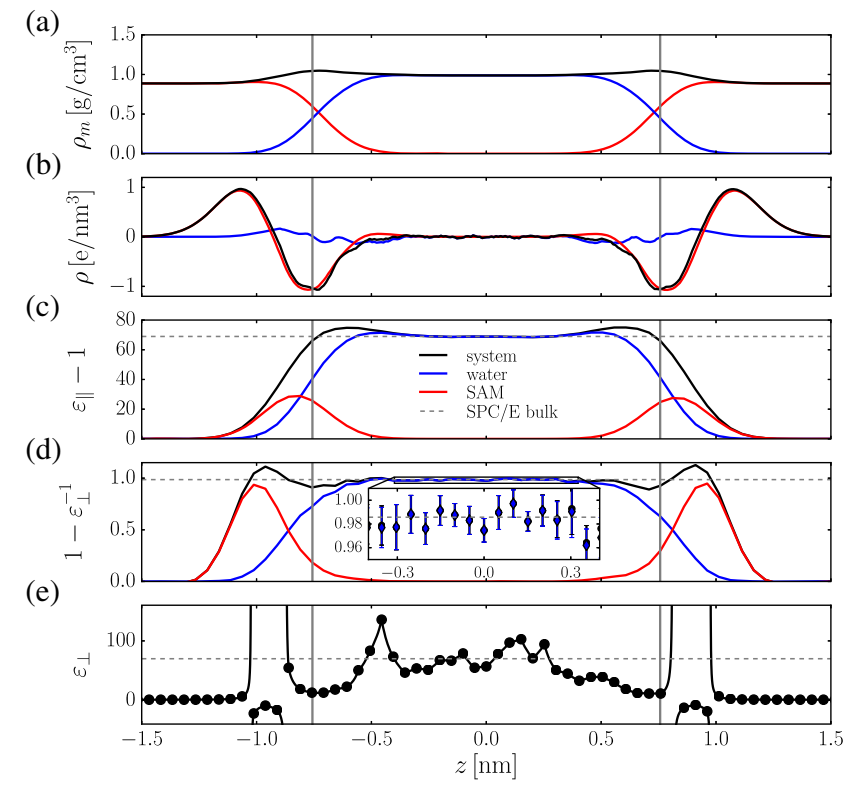

FIG. 2. MD results for a water slab thickness $L_{w}=1.5 \mathrm{~nm}$. Profiles for (a) mass density, (b) charge density, (c) parallel dielectric response, (d) inverse perpendicular dielectric response, and (e) perpendicular dielectric response are shown, contributions of water and decanol are indicated by blue and red lines, respectively. The horizontal broken lines denote the bulk value of SPC/E water. The inset in (d) zooms into the slab central region where bulk water behavior is obtained. The vertical gray lines indicate the Gibbs dividing surface positions. and is negative in a finite region. We will see further below that these divergencies are unproblematic for coarsegrained modeling applications. The inset in Fig. 2(d) demonstrates that $1-\varepsilon_{\perp}^{-1}(z)$ in the slab center approaches the expected bulk value $1-1 / 70=0.986$ with high precision. Not surprisingly, the data for $\varepsilon_{\perp}(z)$ in Fig. 2(e) show significant numerical noise (error bars larger than figure height) but are consistent with the expected bulk value $\varepsilon_{\text {bulk }}=70$ (denoted by a horizontal broken line) in the slab middle. As this shows, the inverse relation between $c_{\perp}(z)$ and $\varepsilon_{\perp}(z)$ in Eq. (6) makes the estimation of the perpendicular dielectric profile in thin slabs numerically highly demanding.

Self and collective polarizations.-Using that the total polarization can be written as $M_{\alpha}=\sum_{i} p_{i, \alpha}$, where $p_{i}$ is the dipole moment of molecule $i$, we split the polarization variance $C_{\alpha}=C_{\alpha}^{\text {self }}+C_{\alpha}^{\text {coll }}$ into self and collective contributions defined as

$$
\begin{gathered}
C_{\alpha}^{\text {self }}=\sum_{i}\left\langle p_{i, \alpha}^{2}\right\rangle-\left\langle p_{i, \alpha}\right\rangle^{2} \text { and } \\
C_{\alpha}^{\text {coll }}=\sum_{i} \sum_{j \neq i}\left\langle p_{i, \alpha} p_{j, \alpha}\right\rangle-\left\langle p_{i, \alpha}\right\rangle\left\langle p_{j, \alpha}\right\rangle .
\end{gathered}
$$

The parallel total variance $C_{\|} /\left(A L_{w} \varepsilon_{0} k_{B} T\right)$ in Fig. 3 approaches the bulk value $\varepsilon_{\text {bulk }}-1=69$ (solid line) already for $L_{w}>2 \mathrm{~nm}$; i.e., the parallel polarization fluctuations exhibit bulklike behavior even in thin slabs. In contrast, the perpendicular variance is far from the bulk limit, and based on Eq. (6) is expected to approach the limit $C_{\perp} /\left(A L_{w} \varepsilon_{0} k_{B} T\right)=69$ only at separations larger than hundreds of nanometers, reflecting that electrostatic boundary effects have long-range consequences.

Interestingly, both self-contributions, $C_{\|}^{\text {self }}$ and $C_{\perp}^{\text {self }}$, approach the value for an isolated water molecule in the Langevin model $\varepsilon_{\text {bulk }}^{\text {self }}-1=19$ for $L_{w} \gtrsim 1 \mathrm{~nm}$ [47]. This shows that the single water response is weakly perturbed by correlation effects both in parallel and perpendicular directions. In fact, the slow crossover of $C_{\perp}$ is entirely

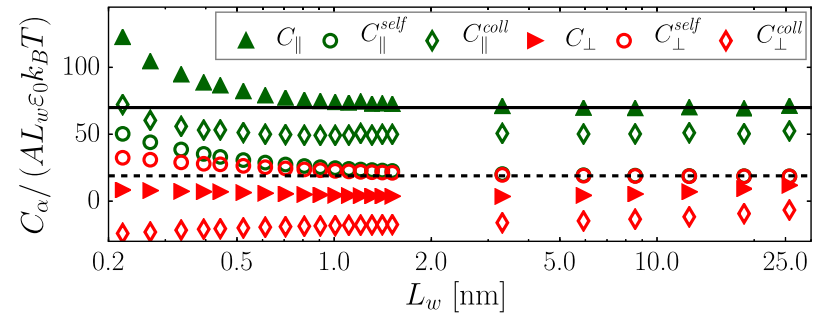

FIG. 3. Total (solid triangles) as well as self (open circles) and collective (open diamonds) polarization variances as a function of water slab thickness $L_{w}$. The solid horizontal line represents the expected bulk limit $C_{\alpha} /\left(A L_{w} \varepsilon_{0} k_{B} T\right)=70-1=69$, the dashed horizontal line shows the Langevin model prediction for an isolated water molecule $C_{\alpha}^{\text {self }} /\left(A L_{w} \varepsilon_{0} k_{B} T\right)=19$. 
(a)

(b)

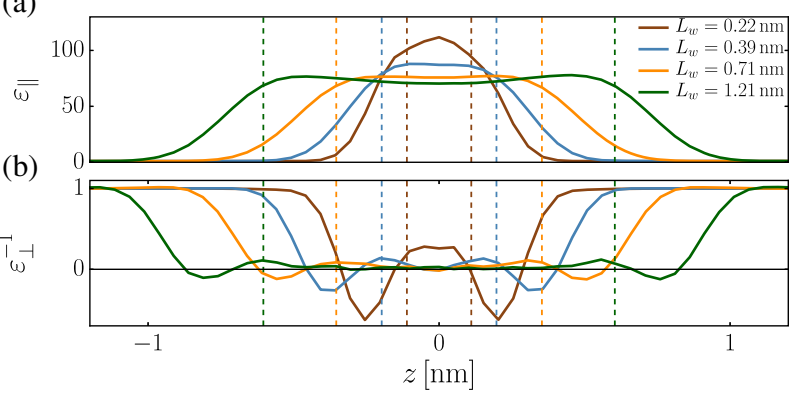

FIG. 4. (a) Parallel and (b) inverse perpendicular dielectric profiles at various water slab thicknesses. The vertical broken lines indicate the corresponding water slab thickness $L_{w}$.

due to the collective contribution $C_{\perp}^{\text {coll }}$, which turns out to be negative for all separations considered by us [red open diamonds in Fig. 3]. This means that the perpendicular water polarizations of neighboring water molecules are anticorrelated.

Dielectric box model.-Figure 4 shows the parallel and inverse perpendicular dielectric profiles for a few different separations. At the smallest separations, $\varepsilon_{\|}(z)$ is enhanced in the slab center while $\varepsilon_{\perp}^{-1}(z)$ is negative over an extended range. To cast these features into a description suitable for coarse-grained modeling and analytical approaches, we derive a simple dielectric box model based on effective medium theory concepts [62].

Since in the parallel direction $\Delta E_{\|}$is constant, we demand that an effective dielectric box model reproduces the integral over the parallel displacement field, $\int_{-L / 2}^{L / 2} d z \Delta D_{\|}(z)$. Assuming an effective dielectric constant $\varepsilon_{\|}^{\text {eff }}$ in a box of width $L^{\text {eff }}$, Eq. (2) leads to

$$
L_{\|}^{\mathrm{eff}}\left(\varepsilon_{\|}^{\mathrm{eff}}-1\right)+L_{z}=\int_{-L_{z} / 2}^{L_{z} / 2} \varepsilon_{\|}(z) d z
$$

In the perpendicular direction $\Delta D_{\perp}$ is constant; thus, we demand the effective box model to reproduce the integral

(a)

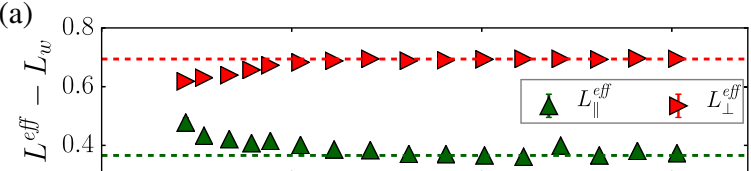

(b)

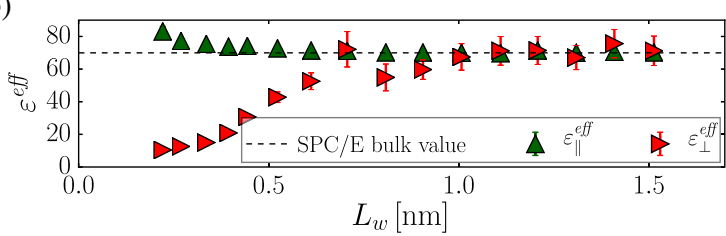

FIG. 5. (a) Shifts of the effective dielectric box widths $L_{\alpha}^{\text {eff }}-$ $L_{w}$ assuming effective dielectric constants corresponding to the bulk value as defined by Eqs. (10) and (11). The horizontal dashed lines denote the asymptotic shifts $\delta_{\alpha}=L_{\alpha}^{\text {eff }}-L_{w}$ for large separation. (b) Effective dielectric constants $\varepsilon_{\alpha}^{\text {eff }}$ when assuming effective box widths given by $L_{\alpha}^{\text {eff }}=L_{w}+\delta_{\alpha}$. over the electric field, $\int_{-L / 2}^{L / 2} d z \Delta E_{\perp}(z)$, which is nothing but the electrostatic potential difference at the system boundaries. Using Eq. (3), we obtain

$$
\int_{-L_{z} / 2}^{L_{z} / 2} d z \varepsilon_{\perp}^{-1}(z)=L_{\perp}^{\mathrm{eff}}\left(\frac{1}{\varepsilon_{\perp}^{\mathrm{eff}}}-1\right)+L_{z}
$$

Obviously, Eqs. (10) and (11) depend on two unknowns each, the box width $L_{\alpha}^{\text {eff }}$ and the effective dielectric constant $\varepsilon_{\alpha}^{\text {eff }}$. In Figure 5(a) we show $L_{\alpha}^{\text {eff }}-L_{w}$ as a function of the water slab thickness $L_{w}$ when assuming $\varepsilon_{\alpha}^{\text {eff }}=\varepsilon_{\text {bulk }}$. For both components we observe $L_{\alpha}^{\text {eff }}-L_{w}>0$, meaning that water slabs at polar surfaces possess a positive dielectric interfacial excess that we associate with contributions from polar orientable headgroups. For the parallel component, the effective box width increases at small separations, indicating that confined water becomes a more efficient parallel dielectric. In contrast, $L_{\perp}^{\text {eff }}$ decreases for small $L_{w}$; thus, the perpendicular dielectric efficiency of confined water decreases. The shifts $L_{\alpha}^{\text {eff }}-L_{w}$ saturate for large $L_{w}$ at $L_{\|}^{\text {eff }}-L_{w} \equiv \delta_{\|}=0.4 \mathrm{~nm}$ and $L_{\perp}^{\text {eff }}-L_{w} \equiv \delta_{\perp}=0.7 \mathrm{~nm}$, indicated by dashed lines in Fig. 5(a). Using now for the effective box widths the asymptotic results for large surface separation $L_{\alpha}^{\text {eff }}=L_{w}+\delta_{\alpha}$, Fig. 5(b) shows the resulting effective dielectric constants. $\varepsilon_{\|}^{\text {eff }}$ increases for small separations and reaches a value of about $\varepsilon_{\|}^{\text {eff }}=85$ at the smallest separation. In contrast, $\varepsilon_{\perp}^{\text {eff }}$ drastically decreases for $D<1 \mathrm{~nm}$ and saturates at about $\varepsilon_{\perp}^{\text {eff }}=10$ at the smallest separation. We thus predict that electrostatic interactions between surfaces, which are described by the perpendicular dielectric component, will be strongly enhanced at small separations.

While we expect details of the dielectric profiles to depend on the surface stiffness and the specific surface chemistry, preliminary simulation results for different surface types suggest the decrease of the perpendicular dielectric component for small separations to be a rather universal feature. Since subnanometer water layers are typical for strongly compressed surfaces in technological and biological applications, our results have consequences for a number of applications. In particular, we suggest that part of the deviations of experimentally measured interactions between charged surfaces from standard PoissonBoltzmann predictions, that are typically ascribed to hydration interactions [63], might in fact be due to a decreasing perpendicular dielectric constant at strong confinement.

We acknowledge the DFG for funding via the SFB 1078.

*aschlaich@physik.fu-berlin.de †netz@physik.fu-berlin.de

[1] J. Israelachvili and H. Wennerström, Nature (London) 379, 219 (1996). 
[2] Q. Du, E. Freysz, and Y. R. Shen, Phys. Rev. Lett. 72, 238 (1994).

[3] B. W. Ninham, Adv. Colloid Interface Sci. 83, 1 (1999).

[4] P. W. Snyder, M. R. Lockett, D. T. Moustakas, and G. M. Whitesides, Eur. Phys. J. Spec. Top. 223, 853 (2014).

[5] J. Israelachvili and G. Adams, J. Chem. Soc., Faraday Trans. 1 74, 975 (1978).

[6] U. Raviv and J. Klein, Science 297, 1540 (2002).

[7] S. Leikin, V. A. Parsegian, D. C. Rau, and R. P. Rand, Annu. Rev. Phys. Chem. 44, 369 (1993).

[8] A. Pertsin, D. Platonov, and M. Grunze, Langmuir 23, 1388 (2007).

[9] C. Eun and M. L. Berkowitz, J. Phys. Chem. B 113, 13222 (2009).

[10] N. Shenogina, R. Godawat, P. Keblinski, and S. Garde, Phys. Rev. Lett. 102, 156101 (2009).

[11] E. Schneck, F. Sedlmeier, and R. R. Netz, Proc. Natl. Acad. Sci. U.S.A. 109, 14405 (2012).

[12] M. Kanduč, A. Schlaich, E. Schneck, and R. R. Netz, Adv. Colloid Interface Sci., 208, 142 (2014).

[13] S. McLaughlin, Annu. Rev. Biophys. Biophys. Chem. 18, 113 (1989).

[14] D. F. Evans and H. Wennerström, The Colloidal Domain: Where Physics, Chemistry, Biology, and Technology Meet (Wiley, New York, 1999).

[15] O. Stern, Z. Elektrochem. Angew. Phys. Chem. 30, 508 (1924).

[16] J. Lyklema and M. Minor, Colloids Surf. A 140, 33 (1998).

[17] L. Herrmann, A. Johner, and P. Kékicheff, Phys. Rev. Lett. 113, 268302 (2014).

[18] A. Abrashkin, D. Andelman, and H. Orland, Phys. Rev. Lett. 99, 077801 (2007).

[19] S. Buyukdagli, M. Manghi, and J. Palmeri, Phys. Rev. E 81, 041601 (2010).

[20] V. Jadhao, F. J. Solis, and M. O. de la Cruz, Phys. Rev. Lett. 109, 223905 (2012).

[21] R. F. Loring and S. Mukamel, J. Chem. Phys. 87, 1272 (1987).

[22] P. A. Bopp, A. A. Kornyshev, and G. Sutmann, Phys. Rev. Lett. 76, 1280 (1996).

[23] K. M. Dyer, J. S. Perkyns, G. Stell, and B. M. Pettitt, Mol. Phys. 107, 423 (2009).

[24] A. Levy, D. Andelman, and H. Orland, Phys. Rev. Lett. 108, 227801 (2012).

[25] V. Ballenegger and J.-P. Hansen, J. Chem. Phys. 122, 114711 (2005).

[26] D. J. Bonthuis, S. Gekle, and R. R. Netz, Phys. Rev. Lett. 107, 166102 (2011).

[27] A. C. Maggs and R. Everaers, Phys. Rev. Lett. 96, 230603 (2006).

[28] S. Buyukdagli and R. Blossey, J. Chem. Phys. 140, 234903 (2014).

[29] D. J. Bonthuis and R. R. Netz, Langmuir 28, 16049 (2012).

[30] S. Senapati and A. Chandra, J. Phys. Chem. B 105, 5106 (2001).

[31] J. Faraudo and F. Bresme, Phys. Rev. Lett. 92, 236102 (2004).

[32] A. Ghoufi, A. Szymczyk, R. Renou, and M. Ding, Europhys. Lett. 99, 37008 (2012).

[33] V. A. Froltsov and S. H. L. Klapp, J. Chem. Phys. 126, 114703 (2007).
[34] S. Gekle and A. Arnold, Phys. Rev. Lett. 111, 089801 (2013).

[35] H. Zhu, A. Ghoufi, A. Szymczyk, B. Balannec, and D. Morineau, Phys. Rev. Lett. 111, 089802 (2013).

[36] M. González-Melchor, E. Mayoral, M. E. Velázquez, and J. Alejandre, J. Chem. Phys. 125, 224107 (2006).

[37] A. Moshfegh and A. Jabbarzadeh, Microfluid. Nanofluid. 20, 67 (2016).

[38] H. A. Stern and S. E. Feller, J. Chem. Phys. 118, 3401 (2003).

[39] M. Neumann, Mol. Phys. 50, 841 (1983).

[40] H. J. C. Berendsen, J. R. Grigera, and T. P. Straatsma, J. Phys. Chem. 91, 6269 (1987).

[41] M. Rami Reddy and M. Berkowitz, Chem. Phys. Lett. 155, 173 (1989).

[42] C. Oostenbrink, A. Villa, A. E. Mark, and W. F. Van Gunsteren, J. Comput. Chem. 25, 1656 (2004).

[43] M. Kanduč, E. Schneck, and R. R. Netz, Chem. Phys. Lett. 610-611, 375 (2014).

[44] M. J. Abraham, T. Murtola, R. Schulz, S. Páll, J. C. Smith, B. Hess, and E. Lindahl, SoftwareX 1-2, 19 (2015).

[45] N. Michaud-Agrawal, E. J. Denning, T. B. Woolf, and O. Beckstein, J. Comput. Chem. 32, 2319 (2011).

[46] V. Parsegian, N. Fuller, and R. Rand, Proc. Natl. Acad. Sci. U.S.A. 76, 2750 (1979).

[47] See Supplemental Material at http://link.aps.org/ supplemental/10.1103/PhysRevLett.117.048001, which includes Refs. [46,48-56], for details on the simulation method, thermodynamic extrapolation, and additional figures and calculations.

[48] B. Widom, J. Chem. Phys. 39, 2808 (1963).

[49] M. P. Allen and D. J. Tildesley, Computer Simulation of Liquids (Oxford University Press, New York, 1989).

[50] D. Frenkel and B. Smit, Understanding Molecular Simulation: From Algorithms to Applications (Academic Press, New York, 2001).

[51] M. R. Shirts and J. D. Chodera, J. Chem. Phys. 129, 124105 (2008).

[52] R. Rand and V. Parsegian, Biochim. Biophys. Acta 988, 351 (1989).

[53] J. N. Israelachvili and R. M. Pashley, Nature (London) 306, 249 (1983).

[54] J. Marra and J. Israelachvili, Biochemistry 24, 4608 (1985).

[55] R. M. Pashley, J. Colloid Interface Sci. 83, 531 (1981).

[56] H. J. C. Berendsen, J. P. M. Postma, W. F. v. Gunsteren, A. DiNola, and J. R. Haak, J. Chem. Phys. 81, 3684 (1984).

[57] U. Essmann, L. Perera, M. L. Berkowitz, T. Darden, H. Lee, and L. G. Pedersen, J. Chem. Phys. 103, 8577 (1995).

[58] D. J. Bonthuis, S. Gekle, and R. R. Netz, Langmuir 28, 7679 (2012).

[59] C. D. Lorenz, J. M. D. Lane, M. Chandross, M. J. Stevens, and G. S. Grest, Langmuir 25, 4535 (2009).

[60] L. Ramin and A. Jabbarzadeh, Langmuir 29, 13367 (2013).

[61] O. Gereben and L. Pusztai, Chem. Phys. Lett. 507, 80 (2011).

[62] J. B. Hasted, Aqueous Dielectrics (Chapman and Hall, London, Distributed in the U.S.A. by Halsted Press, a division of J. Wiley \& Sons, New York, 1973).

[63] V. A. Parsegian, R. P. Rand, and N. L. Fuller, J. Phys. Chem. 95, 4777 (1991). 